\title{
Follicular thyroid carcinoma: differences in clinical relevance between minimally invasive and widely invasive tumors
}

\author{
Mauro Podda, Alessandra Saba, Federica Porru, Isabella Reccia and Adolfo Pisanu*
}

\begin{abstract}
Background: Evidence on the biological behavior and clinical courses of minimally invasive and widely invasive follicular thyroid carcinoma (MI-FTC, WI-FTC) is still debatable. The current study was conducted to identify differences between $\mathrm{Ml}$ and $\mathrm{WI}$ tumors and those prognostic parameters influencing late outcome such as local recurrence and survival.

Methods: From January 1998 to October 2013, 71 patients were operated on in our department because of a FTC. A retrospective cohort study was carried out to compare 42 MI-FTC and 29 WI-FTC. The comparison involved evaluation of patient characteristics, tumor characteristics, tumor staging, and risk assessment.

Results: A diameter greater than $4.0 \mathrm{~cm}$, the presence of vascular invasion, the TNM stage III-IVA, and the high risk at AMES system risk stratification were independent factors significantly related to the presence of a WI-FTC. The only independent predictor of recurrence and disease-free survival at 10-year follow-up was a tumor size greater than $4.0 \mathrm{~cm}$.
\end{abstract}

Conclusions: More attention must be paid in the postoperative tumor re-staging of those patients with tumor size larger than $4.0 \mathrm{~cm}$, which was the only parameter predicting recurrence and influencing disease-free survival. Nevertheless, definitive recommendations cannot be made without a longer follow-up.

Keywords: Follicular thyroid carcinoma, Minimally invasive carcinoma, Widely invasive carcinoma, Lymph node metastasis, Recurrence, Prognosis

\section{Background}

Follicular thyroid carcinoma (FTC) accounts for 10-20 \% of differentiated thyroid carcinomas (DTCs), and it is the second malignant tumor originating from the follicular cells of the thyroid $[1,2]$. In iodine-deficient areas, the relative rate of FTC tends to be higher, up to $40 \%$ of all cases of DTC [3].

According to the World Health Organization (WHO) classification of thyroid tumors, FTC is defined by the presence of capsular and/or vascular invasion and by the absence of nuclear features typical of papillary thyroid carcinoma (PTC) [4]. FTC is more likely to metastasize to distant organs rather than to regional lymph nodes

\footnotetext{
* Correspondence: adolfo.pisanu@unica.it

Department of Surgery, University of Cagliari, Azienda

Ospedaliero-Universitaria, Presidio Policlinico di Monserrato, Blocco G, SS 554 km 4, 500 - 09042 Monserrato, Cagliari, Italy
}

because of its tendency to invade blood vessels thus resulting in hematogenous dissemination [5].

WHO classification also divides FTC into minimally invasive follicular thyroid carcinoma (MI-FTC) when limited capsular and/or vascular invasion is found and widely invasive follicular thyroid carcinoma (WI-FTC) in the case of widespread infiltration of thyroid tissue and/or vascular invasion [4]. Several authors usually reported a benign clinical course of MI-FTC owing to the low risk of tumor recurrence and distant dissemination [1, 6-10]. Conversely, other authors described that also MI-FTCs can give rise to distant metastases [11].

WI-FTC is deemed to have a worse prognosis than MI-FTC [12, 13]. The subset of prognostic factors associated with WI-FTC, however, has not been deeply investigated yet [2]. 
Moreover, the impact of vascular invasion on prognosis is still a matter to be debated as some authors further subdivided MI-FTCs into angioinvasive and non-angioinvasive tumors $[11,14]$.

Age older than 45, sex, extrathyroid invasion, greater tumor size, and the presence of distant metastasis at presentation are recognized risk factors for poorer prognosis of FTC $[5,12,15,16]$. Nevertheless, there is controversy concerning the prognostic significance of these risk factors, which could influence the treatment decision-making of patients with FTC [11].

As evidence on the biological behavior and clinical courses of MI-FTC and WI-FTC is still debatable, we carried out a retrospective cohort study in order to identify differences between MI and WI tumors and those prognostic parameters influencing late outcome such as recurrence and distant metastases. The current research was based on a single-institution experience, with homogeneity in the clinical management of patients with FTC.

\section{Methods}

From January 1998 to October 2013, 556 consecutive patients were operated on in our surgical department because of a DTC. Among them, 71 (12.7 \%) were patients with FTC and they represented the cohort of patients for the current study.

FTC was defined in accordance with the 2004 WHO classification of thyroid tumors as an invasive neoplasm of follicular cell origin without the typical nuclear features of PTC. Tumors were divided into MI-FTC when limited capsular and/or vascular invasion was found and WI-FTC in the case of widespread infiltration of thyroid tissue and/or blood vessels [4]. Tumors with $\leq 3$ foci of vascular invasion were classified as MI-FTCs, whilst tumors with $>3$ foci of vascular invasion were classified as WI-FTCs. Hürthle cell thyroid tumors were excluded from the study as we consider oncocytic cell tumor as a distinctive clinical entity [17]. The follicular variant of papillary thyroid cancer was also excluded.

All patients underwent ultrasonography of both thyroid and neck before operation. Those patients with suspect of lymph node involvement by the tumor at ultrasonography underwent magnetic resonance imaging (MRI) of the neck and mediastinum.

Fine-needle aspiration cytology (FNAC) samples of the thyroid nodules were obtained under ultrasonographic guidance. Thyroid nodules showing cytological microfollicular features with severe atypia were considered as highly suspicious of FTC. The diagnostic categories of cytology were those of the British Thyroid Association (Thy 1, non-diagnostic; Thy 2, benign, non-neoplastic; Thy 3, follicular or suspected follicular or Hürthle neoplasm; Thy 4, suspicious of malignancy; Thy 5, diagnostic of malignancy) [18]. Only a few patients underwent immunocytochemical characterization of follicular nodules by testing the expression of galectin-3 and HBME-1 in FNAC samples. Lateral lymph node involvement was preoperatively assessed by means of both FNAC and/or cytological detection of thyroglobulin in cervical lymph nodes.

Intraoperative frozen section, when executed, was carried out with at least four sections obtained from the tumor. Thin anatomical slices (at least ten) were made to carefully analyze thyroid nodule. Metastatic lymph nodes were routinely examined with four histological sections.

We carried out a retrospective cohort study in order to compare 42 MI-FTC patients and 29 WI-FTC patients. The allocation of patients to the group MI or WI was based on the pathological epicrisis. The current study was conducted in accordance with the recommendations from the Strengthening the Reporting of Observational Studies in Epidemiology (STROBE) guidelines for reporting observational studies [19].

All the medical records were retrospectively reviewed. The comparison involved evaluation of patient characteristics (age and gender), tumor characteristics such as preoperative suspect of malignancy, tumor size, pathologic association, multifocality, thyroid capsular invasion, vascular invasion, extrathyroid invasion, lymph node involvement at presentation, the presence of a well/poorly differentiated tumor, tumor staging, and risk assessment. Pathologic cancer staging was in accordance with the pTNM (pathologic tumor node metastasis) system. Tumor risk was assessed according to the most common risk group system such as AMES (age, metastasis to distant sites, extrathyroidal invasion and size) [20] and AJCC (American Joint Committee on Cancer) pTNM staging following the 2010 pTNMAJCC system [15].

Indications for surgical treatment, management characteristics, surgical complications such as inferior laryngeal nerve palsy and postoperative hypocalcemia, and follow-up results were also evaluated. Postoperative hypocalcemia was defined as a serum calcium level less than $8.0 \mathrm{mg} / \mathrm{dl}$; patients were considered as having permanent hypoparathyroidism and permanent inferior laryngeal nerve palsy if the impaired function persisted after 6 months from the operation [21].

After discharge, all patients entered a scheduled clinical and instrumental follow-up program in the endocrinological department on an average duration of 113.0 months (range 12-288 months) and 125 months (range 24-196 months) in the MI and WI groups, respectively.

All patients diagnosed with a FTC underwent iodine-131 whole-body scan (WBS) 4-6 weeks after surgery, without L-thyroxin treatment and when the TSH level was higher than $30 \mu \mathrm{U} / \mathrm{ml}$. A radioactive-iodine (RAI) ablating dose of $100 \mathrm{mCi}(3700 \mathrm{MBq})$ was used to ablate microscopic 
thyroid remnants and micrometastases. A post-ablating WBS was done to search for missed metastases on the previous WBS. L-thyroxin suppressive treatment was started to maintain a serum TSH concentration of 0.1 $\mu \mathrm{U} / \mathrm{ml}$ or less.

More recently, thyroglobulin (Tg) testing with human recombinant thyrotropin (rhTSH-Thyrogen ${ }^{\circ}$ ) was evaluated in a subgroup of patients prior to radioactive remnant ablation. Patients received $0.9 \mathrm{mg}$ by intramuscular injection on days 1 and 2. Five mCi of iodine-131 were administered orally on day 3 , and a WBS was performed on day 5 . Determination of serum FT3, FT4, Tg, anti-thyroglobulin antibodies, and TSH were performed on day 1 , prior to the first rhTSH injection. Tg and TSH levels were reassessed on days 3,4 , and 5 .

Patients were followed-up at 6 and 12 months after treatment and then on a yearly basis. Only patients with tumor recurrence were resubmitted to RAI ablation therapy. Patients with undetectable TSH-stimulated serum Tg levels, without ultrasonographic evidence of neck lymph node metastases and negative WBS, were defined as cancer-free. Finally, 10-year overall and 10-year disease-free survivals were evaluated in both groups. Written informed consent was obtained from each patient to use data for scientific purposes. The current research was approved and supported by the review board of our Institution and it was conducted in accordance with the Declaration of Helsinki.

\section{Statistical analysis and synthesis of the results}

Data were collected in a planned relational computer database (Microsoft Access ${ }^{\circ}$ ) including patients and tumor characteristics. All statistical analyses were carried out using the MedCalc ${ }^{\circ}$ statistical software version 12.7.5 (MedCalc Software bvba, Ostend, Belgium; http:// www.medcalc.org; 2013).

Data for tumor size and postoperative hospital stay were presented as the mean \pm standard deviation (SD), as the median value and $95 \%$ confidence interval $(\mathrm{CI})$. Data were compared for statistical analysis using the Fisher exact test to evaluate differences between qualitative variables and using the Student's $t$-test to compare quantitative variables. The objective of statistical analysis was also to identify independent risk factors significantly related to the presence of a WI-FTC, to the recurrence of tumors, and to the diseasefree survival at 10-year follow-up by means of stepwise logistic regression analysis. Differences were considered significant when $p<0.05$. $P$ values of the study have been reported as calculated by the statistical software.

\section{Results}

FTCs represented $12.7 \%$ of all treated DTCs (71/556) during the whole period of the study. MI-FTC group included 36 women and 6 men (at a ratio of 6.0:1.0) with a mean age of 45.74 years (range $20-78$ years). WI-FTC group consisted of 26 women and 3 men (at a ratio of 8.6:1.0) with a mean age of 48.48 years (range 19-75 years). Age and gender were not significantly different between groups. Moreover, the distribution of patients over and under 45 was similar in both groups.

The comparative cohort study revealed that there was no statistically significant difference regarding preoperative cytological diagnosis between groups. As shown in Table 1, 30 patients $(71.4 \%)$ with MI-FC and 15 patients (51.7\%) with WI-FTC were preoperative suspected as malignant by cytology and for this reason, total thyroidectomy was performed as a scheduled procedure. Altogether, the follicular neoplasm of 26 patients was characterized by immunocytochemical expression of galectin-3 and HBME-1 on FNAC sample, without significant difference between MI-FTC and WI-FTC (data not shown in tables). Five patients of the WI-FTC group were diagnosed with cervical lymph node

Table 1 Tumor characteristics

\begin{tabular}{|c|c|c|c|}
\hline Characteristics & MI-FTC & WI-FTC & $p$ \\
\hline $\mathrm{N}^{\circ}$ & 42 & 29 & - \\
\hline $\begin{array}{l}\text { Suspect of malignancy } \\
\text { by cytology (pre-op) }\end{array}$ & $30(71.4 \%)^{a}$ & $15(51.7 \%)^{\mathrm{b}}$ & 0.133 \\
\hline Tumor size $(\mathrm{mm})$, mean $\pm \mathrm{SD}$ & $27.05 \pm 11.52$ & $39.34 \pm 15.44$ & 0.000 \\
\hline Range & $6-52$ & $16-75$ & \\
\hline Median (95\% Cl) & $26(23.57-30.53)$ & $38(33.72-44.96)$ & \\
\hline Tumor size $\leq 1.0 \mathrm{~cm}$ & $4(9.5 \%)$ & 0 & 0.140 \\
\hline Tumor size $>1.0 \mathrm{~cm} \leq 2.0$ & $9(21.4 \%)$ & 2 (6.8 \%) & 0.180 \\
\hline Tumor size $>2.0 \mathrm{~cm} \leq 4.0$ & $25(59.6 \%)$ & $15(51.8 \%)$ & 0.628 \\
\hline Tumor size $>4 \mathrm{~cm}$ & $4(9.5 \%)$ & $12(41.4 \%)$ & 0.003 \\
\hline \multicolumn{4}{|l|}{ Pathologic association } \\
\hline Multinodular goiter & 13 (30.9 \%) & $10(34.6 \%)$ & 0.800 \\
\hline Hashimoto's thyroiditis & $13(30.9 \%)$ & $5(17.2 \%)$ & 0.269 \\
\hline Graves' disease & $1(2.4 \%)$ & 0 & 0.851 \\
\hline Normal thyroid & $15(35.8 \%)$ & $14(48.2 \%)$ & 0.332 \\
\hline Follicular thyroid adenoma & 1 (2.4\%) & 0 & 0.851 \\
\hline Papillary microcarcinoma & $6(14.3 \%)$ & $3(10.3 \%)$ & 0.729 \\
\hline Multifocality & 0 & $2(6.8 \%)$ & 0.163 \\
\hline Thyroid capsular invasion & 0 & $1(3.4 \%)$ & 0.408 \\
\hline Vascular invasion & $9(21.4 \%)$ & $15(51.8 \%)$ & 0.011 \\
\hline Extra thyroid invasion & 0 & 1 (3.4\%) & 0.408 \\
\hline Lymph node metastasis & 0 & $5(17.2 \%)$ & 0.009 \\
\hline Well/Poorly differentiated & 0 & $2(6.8 \%)$ & 0.163 \\
\hline M1 at diagnosis & 0 & 0 & - \\
\hline $\begin{array}{l}\text { Concomitant parathyroid } \\
\text { cancer }\end{array}$ & $1(2.4 \%)$ & 0 & 0.851 \\
\hline
\end{tabular}

MI-FTC minimally invasive - follicular thyroid carcinoma, WI-FTC widely invasive - follicular thyroid carcinoma, SD standard deviation, Cl confidence interval, M1 metastasis to distant sites

${ }^{a}$ MI-FTC: 28 Thy 3, 2 Thy 4

bWI-FTC: 13 Thy 3, 2 Thy 4 
metastasis. In this subgroup, cytological diagnosis of cervical lymph nodes was made in four patients, whilst high levels of lymph node thyroglobulin were detected in one patient (data not shown in tables).

Mean tumor size was significantly greater in the WI-FTC group than in the MI-FTC group (39.34 vs. $27.05 \mathrm{~mm}, p=0.000)$. Indeed, WI-FTCs were significantly found with a diameter greater than $4.0 \mathrm{~cm} \quad(p=0.003)$ when compared to MI-FTCs. There was no statistically significant difference regarding pathologic thyroid association. At univariate analysis, vascular invasion and lymph node involvement at presentation were significantly associated to the presence of a WI-FTC ( $p=0.011$ and $p=0.009$, respectively) (Table 1 ).

Higher cancer stage (III-IVA) was significantly related to the presence of a WI-FTC $(p=0.006)$. High risk of the AMES classification was significantly found in the WI-FTC group $(p=0.023)$ (Table 2). Patients of both groups were $\mathrm{M} 0$ at presentation.

Operative results, postoperative morbidity, and data about postoperative ablation therapy with iodine-131 have been reported in details in Table 3. Five patients of the WI-FTC group underwent total thyroidectomy associated with neck lymphadenectomy, whilst no patients of the MI-FTC group had more than total thyroidectomy. There was no difference between groups about postoperative hypocalcemia and postoperative laryngeal nerve palsy. None of the patients of this cohort had permanent hypoparathyroidism, and one patient of the WI-FTC group had permanent inferior laryngeal nerve palsy.

After surgical treatment, all MI-FTC patients were submitted to a standard dose of $100 \mathrm{mCi}$ of RAI ablation therapy. During follow-up, an additional dose of RAI ablation therapy was administered in five patients because of high thyroglobulin levels $(>10 \mathrm{ng} / \mathrm{ml})$ without evidence of tumor recurrence. All WI-FTC patients were submitted to a standard dose of $100 \mathrm{mCi}$ of RAI ablation therapy. During follow-up, an additional dose of RAI ablation therapy was administered in one patient because

Table 2 Tumor staging and risk assessment

\begin{tabular}{llll}
\hline Classification & MI-FTC & WI-FTC & $p$ \\
\hline No & 42 & 29 & - \\
pTNM - AJCC Staging 2010 & & & \\
I & $23(54.8 \%)$ & $11(37.9 \%)$ & 0.024 \\
II & $13(30.9 \%)$ & $4(13.8 \%)$ & 0.156 \\
III & $6(14.3 \%)$ & $13(44.9 \%)$ & 0.006 \\
IVA & 0 & $1(3.4 \%)$ & 0.408 \\
AMES & & & \\
Low risk & $32(76.2 \%)$ & $14(48.2 \%)$ & \\
High risk & $10(23.8 \%)$ & $15(51.8 \%)$ & 0.023 \\
\hline
\end{tabular}

MI-FTC minimally invasive - follicular thyroid carcinoma, WI-FTC widely invasive - follicular thyroid carcinoma
Table 3 Operative technique and postoperative results

\begin{tabular}{|c|c|c|c|}
\hline Parameter & MI-FTC & WI-FTC & $p$ \\
\hline $\mathrm{N}^{\circ}$ & 42 & 29 & \\
\hline$\pi$ & $40(95.2 \%)$ & $21(72.6 \%)$ & \\
\hline Completion thyroidectomy & $2(4.8 \%)$ & $3(10.4 \%)$ & \\
\hline$\pi+C N D$ & - & $2(6.8 \%)$ & \\
\hline$\pi+C N D+M R N D$ ipsilateral & - & $1(3.4 \%)$ & \\
\hline$\Pi+C N D+$ MRND bilateral & - & $2(6.8 \%)$ & \\
\hline Frozen section (true positive) & $2(4.8 \%)$ & 0 & \\
\hline Frozen section (false negative) & $2(4.8 \%)$ & $1(3.4 \%)$ & \\
\hline \multicolumn{4}{|l|}{ Mean postoperative hospital stay } \\
\hline (days) $\pm S D$ & $3.7 \pm 2.0$ & $4.1 \pm 2.5$ & 0.520 \\
\hline Median $(95 \% \mathrm{Cl})$ & $3(3.1-4.3)$ & $3(3.1-5.0)$ & \\
\hline \multicolumn{4}{|l|}{ Morbidity } \\
\hline Transient hypocalcaemia & $6(14.2 \%)$ & $4(13.8 \%)$ & 0.773 \\
\hline Permanent hypocalcemia & - & - & \\
\hline $\begin{array}{l}\text { Transient unilateral laryngeal } \\
\text { nerve palsy }\end{array}$ & - & - & \\
\hline $\begin{array}{l}\text { Permanent laryngeal nerve } \\
\text { palsy }\end{array}$ & - & $1(3.4 \%)$ & 0.851 \\
\hline \multicolumn{4}{|l|}{ I131 ablation therapy } \\
\hline 0 application & - & - & - \\
\hline 1 application & $37(88.1 \%)$ & $22(76.0 \%)$ & 0.209 \\
\hline 2 applications & $5(11.9 \%)$ & $4(13.8 \%)$ & 0.898 \\
\hline 4 applications & - & $1(3.4 \%)$ & 0.064 \\
\hline 5 applications & - & 1 (3.4\%) & \\
\hline 8 applications $^{\mathrm{a}}$ & - & $1(3.4 \%)$ & \\
\hline Tumor recurrence (node) & - & $3(10.4 \%)$ & 0.064 \\
\hline Lateral neck compartment & - & $3(10.4 \%)$ & \\
\hline Central neck compartment & - & - & \\
\hline Loco-regional & - & 1 (3.4\%) & \\
\hline M1 at follow-up & - & $3(10.4 \%)$ & 0.064 \\
\hline Overall recurrence rate & - & $7(24.1 \%)$ & 0.001 \\
\hline Death of thyroid cancer & - & - & \\
\hline Death of other causes & - & - & \\
\hline Mean follow-up duration & $113(12-288)$ & $125(24-196)$ & \\
\hline
\end{tabular}
(months) (range)

MI-FTC minimally invasive - follicular thyroid carcinoma, WI-FTC widely invasive - follicular thyroid carcinoma, CND central neck dissection, MRND modified radical neck dissection, $S D$ standard deviation, $C l$ confidence interval, $M 1$ metastasis to distant site

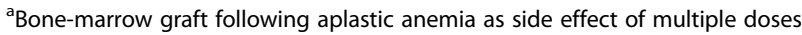
of RAl ablation therapy

of high thyroglobulin levels without evidence of tumor recurrence; three patients had an additional dose of RAI ablation after removal of nodal recurrence in the lateral neck compartment $(15,24$, and 25 months after the operation, respectively); two patients had distant metastasis at 12 and 36 months after surgery, and they underwent a total of four and five doses of RAI ablation therapy, 
respectively. Finally, one patient with bilateral lymph node involvement had nodal and loco-regional recurrence 15 months after surgery and she was once more submitted to a dose of $100 \mathrm{mCi}$ of RAI ablation therapy. Afterwards, she underwent a total of eight doses of RAI ablation therapy for the occurrence of distant metastases. At 156 months from the first operation, she is alive but she is not disease-free. Moreover, she was submitted to a bone-marrow graft following an aplastic anemia as side effect of multiple doses of RAI ablation therapy.

When analyzing seven patients with recurrence, three of them underwent total thyroidectomy and neck lymphadenectomy at first operation. In these patients, recurrence was found in cervical nodes, which were surgically removed before the second RAI ablation cycle. One more patient, who previously underwent total thyroidectomy alone, had a cancer recurrence in the thyroid bed and he was treated by only RAI ablation. Finally, three patients with distant metastases were treated by multiple doses of RAI ablation.

After a mean follow-up of 103.0 and 125.0 months in the MI and WI group, respectively, none of the patients in either group died because of FTC. Patients of the MI-FTC group had $100 \%$ overall and disease-free survival at 10 years. Patients of the WI-FTC group had $100 \%$ overall survival and $75.8 \%$ disease-free survival at 10 years, respectively.

Multivariate analysis showed that a diameter $>4 \mathrm{~cm}$, the presence of vascular invasion, the TNM stage III-IVA and the high risk of AMES system risk stratification were independent prognostic factors significantly related to the presence of a WI-FTC (Table 4).

Tumor size larger than $4.0 \mathrm{~cm}$ was the only factor retained in the multivariate statistical model as independent predictor of overall recurrence and influencing disease-free survival (Table 5).

\section{Discussion}

Pathological diagnosis, treatment policy, and prognosis of both MI-FTC and WI-FTC are still matters to be debated. Recently, the review of the European Society of Endocrine Surgeons has been published in an effort to reach an evidence-based consensus on the management

Table 4 Prognostic factors significantly related to the presence of a widely invasive FTC

\begin{tabular}{lllllll}
\hline Parameter & $\beta$ & SE & $\chi^{2}$ & $p$ & OR & $95 \% \mathrm{Cl}$ \\
\hline Diameter $>4 \mathrm{~cm}$ & 1.758 & 0.650 & 8.306 & 0.006 & 5.805 & $1.632-20.764$ \\
Vascular invasion & 1.368 & 0.528 & 7.027 & 0.009 & 3.928 & $1.393-11.072$ \\
Stage III-IVA & 1.722 & 0.576 & 9.807 & 0.002 & 5.600 & $1.808-17.340$ \\
AMES High Risk & 1.232 & 0.519 & 5.848 & 0.017 & 3.428 & $1.239-9.481$ \\
\hline
\end{tabular}

Stepwise logistic regression analysis

$\beta$ coefficient, $S E$ standard error, $X^{2}$ chi square, $O R$ odds ratio, $\mathrm{Cl}$ confidence interval, FTC follicular thyroid carcinoma
Table 5 Independent risk factors for overall recurrence and disease-free survival after stepwise logistic regression analysis

\begin{tabular}{lllllll}
\hline Parameter & $\beta$ & SE & $x^{2}$ & $p$ & OR & $95 \% \mathrm{Cl}$ \\
\hline Diameter $>4 \mathrm{~cm}$ & 1.909 & 0.967 & 3.903 & 0.048 & 6.750 & $1.01-44.92$ \\
\hline$\beta$ coffict, SE stand
\end{tabular}

$\beta$ coefficient, $S E$ standard error, $X^{2}$ chi square, $O R$ odds ratio, $C I$ confidence interval

of MI-FTC [22], whilst Ito et al. reported the clinical significance of specific prognostic factors of WI-FTC [2]. Within this regard, the current study was carried out in order to identify differences in clinical relevance between MI and WI tumors.

The main results of the current investigation showed that a diameter greater than $4.0 \mathrm{~cm}$, the presence of vascular invasion, the TNM stage III-IVA, and the high risk at AMES system risk stratification were independent factors significantly related to the presence of a WI-FTC. The only factor retained in the multivariate statistical model as independent predictor of overall recurrence and disease-free survival at 10-year follow-up was a tumor size greater than $4.0 \mathrm{~cm}$. Moreover, MI-FTC group showed a median diameter of $2.6 \mathrm{~cm}$ vs. $3.8 \mathrm{~cm}$ of the WI one and no patients of the MI group presented with tumor recurrence at follow-up.

Our data confirm other studies in which WI-FTCs larger than $4.0 \mathrm{~cm}$ (T3 on TNM staging system) have a worse prognosis because this diameter cut-off significantly affects cancer recurrence and cancer-specific survival $[1,2,23]$. In the case of greater tumors with a more likely aggressive biological behavior, a full treatment protocol including total thyroidectomy, RAI ablation, and L-thyroxin TSH-suppressive therapy and careful follow-up are strongly recommended $[2,16,22]$.

Multivariate analysis showed vascular invasion as independently related to the presence of a WI-FTC. Indeed, the presence of extensive vascular invasion has been reported as associated with a higher risk of relapse in WI-FTCs [22]. Some authors highlighted the importance of vascular invasion in evaluating tumor prognosis by a sub-classification of FTCs. D'Avanzo et al. identified a group of MI-FTCs without vascular invasion (with $98 \%$ of disease-free survival at 10 years), an intermediate MI-FTC group with vascular invasion and with or without capsular invasion (with $80 \%$ of disease-free survival at 10 years), and a WI-FTC group (with $38 \%$ of diseasefree survival at 10 years) [13]. Similarly, O'Neil et al. showed that patients with MI-FTC without vascular invasion had a better disease-free survival at 40 months when compared with patients who had MI-FTC with vascular invasion or WI-FTC $(97,81$, and $46 \%$, respectively) [6]. Several studies suggested that MI-FTC patients without vascular invasion could be treated by a more conservative approach in term of thyroid lobectomy $[6,7,22,24]$. The choice of a less aggressive 
treatment could also be justified by the fact that the overall survival of patients with MI-FTC is essentially identical to that of the general population [25].

Conversely, other studies reported the occurrence of distant metastases also in MI-FTC patients without vascular invasion $[6,11,26]$. This means that MI-FTC could be a clinical dilemma being the most controversial issue to deal with. For these reasons, some authors considered the currently accepted histological classification of FTC as inadequate in predicting aggressive clinical courses [11]. The unclear and sometime unpredictable behaviors of MI-FTC persuaded these authors to recommend total thyroidectomy and RAI ablation for all patients with FTC $[3,11]$. Also in our experience, all patients with MI-FTC were submitted to a full treatment protocol without relapse at 10 year follow-up.

According to other authors, we applied a more aggressive approach in all cases of FTC in terms of total thyroidectomy and RAI ablation therapy to facilitate the oncologic follow-up as well as the detection of occult metastases $[3,6,11]$. Our greatest concern was the possibility of overtreatment of patients belonging to the MI-FTC group. However, in the absence of molecular markers routinely used in predicting different biological behavior of FTC, which is finally diagnosed postoperatively, changing our treatment approach may be difficult.

Moreover, frozen section examination of FTCs has been proposed with the aim of modulating the treatment policy of these neoplasms. Some authors supported the use of frozen section in the management of FTCs because the diagnostic accuracy of malignancy was as high as $78 \%$ [27], whilst several authors did not find any additional diagnostic value when using frozen section in the management of FTCs [28, 29]. In our experience, we performed only five intraoperative frozen sections because three of them were diagnosed as false negative requiring completion thyroidectomy.

Among several prognostic scoring systems, TNM staging is the most commonly used and it is considered as a good system for evaluating FTC $[9,26,30]$. However, the degree of invasiveness such as minimally invasive or widely invasive tumor, which is the worldwide-accepted classification of FTC, is not included in the TNM staging system [10]. Further studies are needed to better assess the impact of this discrepancy in the risk classification of FTC. Moreover, the updated TNM staging system has placed greater emphasis on the metastasis to lymph nodes in predicting prognosis of DTC [15]. In the case of FTC, there is a general agreement that nodal dissection must be performed only with therapeutic intent and in the presence of clinical evidence for lymph node involvement by the tumor $[3,17,22]$. In our practice, we plan neck lymph node dissection only after a preoperative detection of nodal involvement by the tumor (by nodal cytology or high nodal level of Tg). Indeed, when no nodal metastases can be detected preoperatively, there is no indication for prophylactic neck dissection in FTCs [22, 31-33]. Moreover, a significant correlation between tumor sizes of FTC greater than $4.0 \mathrm{~cm}$ and the presence of nodal metastases at presentation was demonstrated in previous studies $[16,33]$.

\section{Conclusions}

The greatest clinical relevance of WI-FTC has been clearly showed by reviewing the literature and our own experience. Within this regard, more attention must be paid in the postoperative tumor re-staging of those patients with tumor size larger than $4.0 \mathrm{~cm}$, which was the only parameter predicting overall recurrence and influencing disease-free survival. However, the unpredictable behaviors of MI-FTC and the need to facilitate the oncologic follow-up persuaded us to recommend a full treatment protocol for all patients with FTC.

An alternative, less aggressive operative strategy, performing intraoperative frozen sections, did not achieve any validation in our practice. Lymphadenectomy in FTCs should be performed only after a preoperative detection of nodal involvement by the tumor.

The good outcomes reported in the current study may be related to our quite aggressive therapeutic strategy. Nevertheless, definitive recommendations cannot be made without a longer follow-up. Finally, the inclusion of the degree of tumor invasiveness in the TNM staging system must be taken into account.

\section{Abbreviations}

AMES: age, metastasis to distant sites, extrathyroidal invasion and size; DTC: differentiated thyroid cancer; FNAC: fine-needle aspiration cytology; FTC: follicular thyroid cancer; HBME-1: Hector Battifora mesothelial cell-1; MI-FTC: minimally invasive - follicular thyroid cancer; MRI: magnetic resonance; RAl: radioactive lodine; Tg: thyroglobulin; TSH: Thyroid-stimulating hormone; WBS: whole body scintigraphy; WI-FTC: widely invasive - follicular thyroid cancer.

\section{Competing interests}

The authors declare that they have no competing interests.

\section{Authors' contributions}

PM did the conception and design, drafting the article, analysis and interpretation of data, revising for intellectual content, approval of the final version. SA did the acquisition of data and analysis, revising for intellectual content, and approval of the final version. PF and RI did the analysis and interpretation of data, revising for intellectual content, and approval of the final version. PA did the conception and design, acquisition and interpretation of data, drafting the article, revising for important intellectual content, and approval of the final version. All authors read and approved the final manuscript.

\section{Acknowledgements}

This study was supported by a grant from the University of Cagliari, Italy (CAR 2013).

Received: 6 January 2015 Accepted: 22 May 2015

Published online: 04 June 2015 


\section{References}

1. Asari R, Koperek O, Scheuba C, Riss P, Kaserer K, Hoffmann M, et al. Follicular thyroid carcinoma in an iodine-replete endemic goiter region: a prospective collected, retrospectively analyzed clinical trial. Ann Surg. 2009;249:1023-31.

2. Ito Y, Hirokawa M, Masuoka H, Yabuta T, Fukushima M, Kihara M, et al. Distant metastasis at diagnosis and large tumor size are significant prognostic factors of widely invasive follicular thyroid carcinoma. Endocr J. 2013;60:829-33.

3. De Crea C, Raffaelli M, Sessa L, Ronti S, Fadda G, Bellantone C, et al. Actual incidence and clinical behavior of follicular thyroid carcinoma: an institutional experience. Sci World J. 2014. article ID 952095, http:// dx.doi.org/10.1155/2014/952095.

4. DeLellis RA, Lloyd RV, Heitz PU, et al. World Health Organization classification of tumors: pathology and genetics of tumors of endocrine organs. Lyon: IARC Press; 2004. p. 64-6. eds.

5. Kim HJ, Sung JY, Oh YL, Kim JH, Son Yl, Min YK, et al. Association of vascular invasion with increased mortality in patients with minimally invasive follicular thyroid carcinoma but not widely invasive follicular thyroid carcinoma. Head Neck. 2013. doi: 10.1002/hed.23511.

6. O'Neill CJ, Vaughan L, Learoyd DL, Sidhu SB, Delbridge LW, Sywak MS Management of follicular thyroid carcinoma should be individualized based on degree of capsular and vascular invasion. Eur J Surg Oncol. 2011;37:181-5.

7. van Heerden JA, Hay ID, Goellner JR, Salomao D, Ebersold JR, Bergstralh EJ, et al. Follicular thyroid carcinoma with capsular invasion alone: a non threatening malignancy. Surgery. 1992:112:1130-6.

8. Huang CC, Hsueh C, Liu FH, Chao TZ, Lin JD. Diagnostic and therapeutic strategies for minimally and widely invasive follicular thyroid carcinomas Surg Oncol. 2011;20:1-6.

9. Lo CY, Chan WF, Lam KY, Wam KY. Follicular thyroid carcinoma: the role of histology and staging systems in predicting survival. Ann Surg. 2005;242:708-15

10. Chow SM, Law SC, Mendenhall WM, Au SK, Yau S, Yuen KT, et al. Follicular thyroid carcinoma: prognostic factors and the role of radioiodine. Cancer. 2002:95:488-98.

11. Ban EJ, Andrabi A, Grodski S, Yeung M, Mclean C, Serpell J. Follicular thyroid cancer: minimally invasive tumors can give rise to metastases. ANZ J Surg. 2012:82:136-9.

12. Lang $B H$, Lo CY, Chan WF, Lam KY, Wan KY. Prognostic factors in papillary and follicular thyroid carcinoma: their implication for cancer staging. Ann Surg Oncol. 2007;14:730-8.

13. D'Avanzo A, Treseler P, Ituarte PH, Wong M, Streja L, Greenspan FS, et al. Follicular thyroid carcinoma: histology and prognosis. Cancer. 2004;100:1123-9.

14. Collini P, Sampietro G, Pilotti S. Extensive vascular invasion is a marker of risk of relapse in encapsulated non-Hürthle cell follicular carcinoma of the thyroid gland: a clinicopathological study of 18 consecutive cases from a single institution with a 11-year median follow up. Histopathology. 2004:44:35-9.

15. Edge SB, Byrd DR, Compton CC. AJCC Cancer Staging Manual, 7th edn, pp 87-96. New York: Springer; 2010.

16. Pisanu A, Deplano D, Pili M, Uccheddu A. Larger tumor size predicts nodal involvement in patients with follicular thyroid carcinoma. Tumori. 2011;97:296-303.

17. Pisanu A, Di Chiara B, Reccia I, Uccheddu A. Oncocytic cell tumors of the thyroid: factors predicting malignancy and influencing prognosis, treatment decisions, and outcomes. World J Surg. 2010;34:836-43.

18. British Thyroid Association, Royal College of Physicians. British Thyroid Association Guidelines for the management of thyroid cancer. 2. 2007. http://www.british-thyroid-association.org/Guidelines/

19. Vandenbroucke JP, von Elm E, Altman DG, Gøtzsche PC, Mulrow CD, Pocock SJ, et al. Strengthening the Reporting of Observational Studies in Epidemiology (STROBE): explanation and elaboration. PLoS Med. 2007:4:e297. doi:10.1371/journal.pmed. 0040297.

20. Cady B, Rossi R, Silverman M, Wool M. Further evidence of the validity of risk group definition in differentiated thyroid carcinoma. Surgery. 1985;98:1171-8.

21. Pisanu A, Reccia I, Nardello O, Uccheddu A. Risk factors for nodal metastasis and recurrence among patients with papillary thyroid microcarcinoma: differences in clinical relevance between nonincidental and incidental tumors. World J Surg. 2009:33:460-8.
22. Dionigi G, Kraimps J-L, Schmid KW, Hermann M, Sheu-Grabellus S-Y, De Wailly $P$, et al. Minimally invasive follicular thyroid cancer (MIFTC) - a consensus report of the European Society of Endocrine Surgeons (ESES). Langenbecks Arch Surg. 2014;399:165-84.

23. Ito Y, Hirokawa M, Higashiyama T, Takamura Y, Miya A, Kobayashi K, et al Prognosis and prognostic factors of follicular carcinoma in Japan: importance of postoperative pathological examination. World J Surg 2007;31:1417-24.

24. Thompson LD, Wieneke JA, Pall E, Frommelt RA, Adair CF, Heffess CS. A clinicopathologic study of minimally invasive follicular carcinoma of the thyroid gland with a review of the English literature. Cancer. 2001;91:505-24.

25. Goffredo P, Cheung K, Roman SA, Sosa JA. Can minimally invasive follicular thyroid carcinoma be approached as a benign lesion? A population-level analysis of survival among 1.200 patients. Ann Surg Oncol. 2013;20:767-72.

26. Sugino K, Kameyama K, Ito K, Nagahama M, Kitagawa W, Shibuya H, et al. Outcomes and prognostic factors of 251 patients with minimally invasive follicular thyroid carcinoma. Thyroid. 2012:22:798-804.

27. Paphavasit A, Thompson GB, Hay ID, Grant CS, van Heerden JA, Ilstrup DM, et al. Follicular and Hürthle cell thyroid neoplasms: is frozen-section evaluation worthwhile? Arch Surg. 1997;132:674-80.

28. Udelsman R, Westra WH, Donovan PI, Sohn TA, Cameron JL. Randomized prospective evaluation of frozen section analysis for follicular neoplasms of the thyroid. Ann Surg. 2001;233:716-22.

29. Bronner MP, Hamilton R, LiVolsi VA. Utility of frozen section on follicular lesions of the thyroid. Endocrinol Pathol. 1994;5:154-61.

30. Rios A, Rodriguez JM, Ferri B, Martinez-Barba E, Febrero B, Parrilla P. Are prognostic scoring systems of value in patients with follicular thyroid carcinoma? Eur J Endocrinol. 2013;169:821-7.

31. Cooper DS, Doherty GM, Haugen BR, Kloos RT, Lee SL, Mandel SJ, et al. American Thyroid Association Guidelines Taskforce: Management guidelines for patient with thyroid nodules and differentiated thyroid cancer. Thyroid. 2006;16:109-41.

32. Pacini $F$, Schlumberger $M$, Dralle $H$, Elisei $R$, Smit JW, Wiersinga W. European Thyroid Cancer Taskforce: European consensus for the management of patients with differentiated thyroid carcinoma of the follicular epithelium. Eur J Endocrinol. 2006;154:787-803.

33. Alfalah $\mathrm{H}$, Cranshaw I, Jany $T$, Arnalsteen L, Leteurtre E, Cardot C, et al. Risk factors for lateral cervical lymph node involvement in follicular thyroid carcinoma. World J Surg. 2008;32:2623-6.

\section{Submit your next manuscript to BioMed Central and take full advantage of:}

- Convenient online submission

- Thorough peer review

- No space constraints or color figure charges

- Immediate publication on acceptance

- Inclusion in PubMed, CAS, Scopus and Google Scholar

- Research which is freely available for redistribution 